\title{
Microtubule-Associated Protein-2: A New Sensitive and Specific Marker for Pulmonary Carcinoid Tumor and Small Cell Carcinoma
}

Yulin Liu, M.D., Charles D. Sturgis, M.D., Dana M. Grzybicki, M.D., Ph.D., Katherine M. Jasnosz, M.D., Peter R. Olson, M.D., Ming Tong, David D. Dabbs, M.D., Stephen S. Raab, M.D., Jan F. Silverman, M.D. Department of Pathology and Laboratory Medicine, Allegheny General Hospital, Pittsburgh, Pennsylvania

Microtubule-associated proteins (MAPs) are a major component of cytoskeleton family proteins associated with microtubule assembly. MAP-2 has been shown to be specifically expressed in neuronally differentiated cells. Pulmonary neuroendocrine carcinomas such as carcinoid tumors and small cell carcinomas are derived from neuroendocrine cells. We hypothesize that neuroendocrine cells may also express MAP-2, and therefore, MAP-2 may be used as a marker for pulmonary carcinomas of neuroendocrine differentiation. To investigate the utility of using MAP-2 expression to separate pulmonary neuroendocrine from nonneuroendocrine tumors, we examined the expression of MAP-2 immunohistochemically in 100 cases of pulmonary carcinomas. The immunoperoxidase method with antigen retrieval was used to characterize the expression of MAP-2, chromogranin, synaptophysin, and neuron-specific enolase in 25 small cell carcinomas, 25 carcinoid tumors, 25 adenocarcinomas, and 25 squamous cell carcinomas. All tumors were lung primaries. All 25 cases of carcinoid tumors $(100 \%)$ as well as 23 of 25 cases $(92 \%)$ of small cell carcinomas were positive for MAP-2. Four of 25 cases ( $16 \%$ ) of adenocarcinomas were positive for MAP-2 and synaptophysin. Among the 25 squamous carcinomas, 4 cases $(16 \%)$ were positive for MAP-2, 2 cases (8\%) were positive for synaptophysin, 11 cases $(44 \%)$ were positive for neuron-specific enolase, and none was positive for chromogranin. In conclusion, MAP-2 is a new sensitive and specific marker for the pulmonary tumors of neuroendocrine differentiation. We recommend that MAP-2 be added to immunohistochemical panels to separate

Copyright (c) 2001 by The United States and Canadian Academy of Pathology, Inc.

VOL. 14, NO. 9, P. 880, 2001 Printed in the U.S.A.

Date of acceptance: May 1, 2001.

Presented at the United States and Canada Academy of Pathology meeting, March 7, 2000, New Orleans, Louisiana.

Address reprint requests to: Yulin Liu, M.D., Department of Pathology and Laboratory Medicine, Allegheny General Hospital, Pittsburgh, PA 15212; e-mail: yliu63@hotmail.com; fax: 412-359-3860. non-neuroendocrine from neuroendocrine lung tumors.

KEY WORDS: Microtubule-associated protein, Neuroendocrine carcinoma, Lung.

Mod Pathol 2001;14(9):880-885

The cytoskeleton is composed of a three-dimensional network assembled from actins, microtubules and intermediate-size filaments. This network is involved in a wide variety of cell functions such as the regulation of cell shape and polarity during cell differentiation and proliferation, intracellular membrane traffic, secretory process, modulation of receptors, spatial organization of cell organelles, chromosome partitioning at mitosis, and intracellular transportation (1, 2). These functions are maintained and supported mainly by the microtubule network that includes tubulin isoforms and microtubule associated proteins (MAPs). Although the tubulin isoforms form the frame of the network, the integrity and intracellular organization of microtubule network is modulated and assembled by MAPs. It is well known that MAPs interact with other cytoskeleton proteins as well $(1,2)$.

Although MAPs have been found to be present in all eukaryotic cells, MAPs have been traditionally isolated and characterized from the brain. The family of MAPs includes high molecular components such as MAP-1A, MAP-1B, MAP-1C, MAP-2A and MAP-2B, the neuronal MAP-3, MAP-4; intermediate size polypeptides such as tau; and small molecular component such as MAP-2C. More than twenty isoforms of neuronal MAPs have been found and characterized during last three decades. MAP-2 is found mainly in dendrites and soma of neuronal cells in most regions of central nervous system. MAP-2 has been used extensively as a very sensitive and specific marker for neuronal differentiation during the last two decades (2).

Most pulmonary carcinomas may be readily classified and characterized based on their morphologic features such as cellular pattern, cell size, nu- 
clear to cytoplasmic ratios and nuclear features such as chromatin pattern and presence or absence of nucleoli. However, the morphologic separation of pulmonary neuroendocrine carcinomas from non-small cell carcinomas may sometimes be difficult and challenging. The distinction between these two groups of carcinomas is very important, because they differ in clinical behavior, prognosis, and treatment, including their response to chemotherapy and irradiation. Although many studies indicate that the immunohistochemical markers for neuroendocrine differentiation such as synaptophysin, chromogranin, Leu-7 and neuron-specific enolase are useful in detecting neuroendocrine differentiation in lung carcinomas, these markers are not specific for neuroendocrine differentiation because positive staining has been reported in nonsmall cell carcinomas (3-11).

Although the cell origin for neuroendocrine carcinomas of the lung is controversial and debatable, most studies support that neuroendocrine carcinomas are derived from Kulchitsky cells, based on the presence of intracytoplasmic dense core granules and immunohistochemical profile $(12,13)$. The utility of MAP-2 expression in neuroendocrine neoplasms has not been investigated. In this study, we compared the sensitivity and specificity of MAP-2 immunoreactivity to that of conventional neuroendocrine markers such as synaptophysin, chromogranin and neuron-specific enolase in neuroendocrine carcinomas and non-small cell carcinomas.

\section{MATERIALS AND METHODS}

Cases were retrieved from the archives of the Department of Pathology and Laboratory Medicine at Allegheny General Hospital from Jan. 1996 to Jan. 1998. A total 100 cases of pulmonary carcinomas were included. The original hematoxylin-eosin stained sections as well as a recut from each case were reviewed to confirm the diagnosis. All tumors were lung primaries based on morphologic finding and chart review. The formalin-fixed paraffin embedded blocks were selected from each case for immunohistochemical studies.

We studied 50 cases of neuroendocrine carcinomas, including 25 cases each of carcinoid tumor and small cell carcinoma and 50 cases of non-small cell carcinomas, consisting of 25 cases of squamous cell carcinoma and 25 cases of adenocarcinoma. Immunostaining was performed using the avidinbiotin-peroxidase complex (ABC) method. Fivemicrometer sections were cut from the paraffin blocks and mounted on charged slides. The sections were deparaffinized, rehydrated, and treated with $1 \%$ hydrogen peroxide for 10 minutes to block endogenous peroxidase activity. Sections then were rinsed and incubated with primary antibodies against MAP-2 (Sigma, St. Louis, MO), chromogranin (Sigma), synaptophysin (SYN; Sigma), and neuron specific enolase (NSE; Sigma). The immunostaining was performed on an automated immunostainer with appropriate positive and negative controls.

\section{RESULTS}

A total of 100 pulmonary carcinomas were studied. The results of the immunoreactive staining are shown in the Table 1. The immunostaining pattern for MAP-2 is diffuse cytoplasmic positivity with no nuclear staining. Strong positive MAP-2 immunoreactivity was seen in carcinoid tumors. The immunoreactivity for synaptophysin, chromogranin, and neuron-specific enolase was also cytoplasmic.

Figure 1 shows the immunoreactivity of MAP-2 for a carcinoid tumor. As shown in Table 1, all 25 cases $(100 \%)$ of carcinoid tumors were strongly and diffusely positive for MAP-2. Twenty-three of 25 cases $(92 \%)$ of small cell carcinomas showed immunoreactivity with MAP-2 (Fig. 2). Unlike diffuse immunoreactivity of MAP-2 seen in carcinoid tumors, MAP-2 immunoreactivity with the small cell carcinomas was weaker and less diffuse. Four cases $(16 \%)$ of poorly differentiated adenocarcinomas showed focal immunoreactivity with MAP-2 and synaptophysin. Two of 25 cases (8\%) of squamous carcinomas were focally positive for MAP-2.

Positive immunoreactivity of synaptophysin was also seen in all 25 cases (100\%) of carcinoid tumors and in 23 cases $(92 \%)$ of small cell carcinomas. The immunoreactivity was diffuse and strong in carcinoid tumors but less diffuse in small cell carcinomas. Four of 25 (16\%) adenocarcinomas and 2 of 25 (8\%) cases of squamous carcinomas were focally positive for synaptophysin.

All 25 cases of carcinoid tumors (100\%) were immunoreactive with chromogranin (Table 1). Twentyone of 25 cases (84\%) of small cell carcinomas were negative for chromogranin. There were 4 cases (16\%) of small cell carcinomas showing focal, weak blush immunoreactivity ( $<5 \%$ of the cells) with chromogranin. None of the 25 cases of adenocarcinomas and 25 cases of squamous carcinomas was immunoreactive with chromogranin.

NSE immunoreactivity was demonstrated in 21 cases $(81 \%)$ of carcinoid tumors and in 10 cases

TABLE 1. The Positive Staining Percentages for MAP-2, SYN, CHR, and NSE

\begin{tabular}{lcccc}
\hline \multicolumn{1}{c}{ Cases } & MAP-2 (\%) & SYN (\%) & CHR (\%) & NSE (\%) \\
\hline Carcinoid tumor & 100 & 100 & 100 & 84 \\
Small-cell carcinoma & 92 & 92 & 0 & 40 \\
Adenocarcinomas & 16 & 16 & 0 & 60 \\
Squamous cell carcinoma & 16 & 8 & 0 & 44 \\
\hline
\end{tabular}

MAP, microtubule-associated protein; SYN, synoptophysin; CHR, chromogranin; NSE, neuron-specific enolase. 

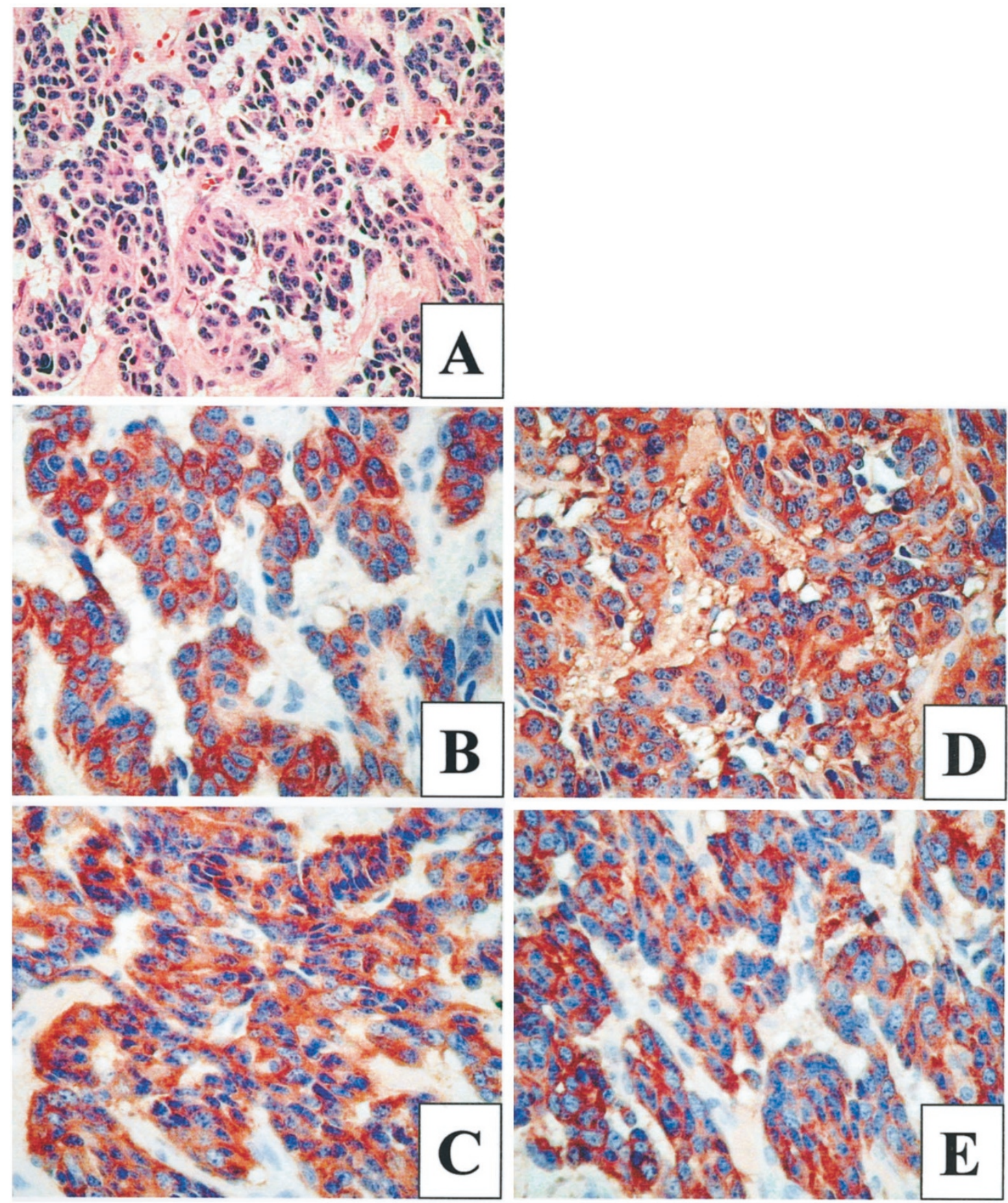

FIGURE 1. Immunoreactivity of microtubule-associated protein-2 (MAP-2), synaptophysin, chromogranin, and neuron-specific enolase (NSE) with carcinoid tumors. A, hematoxylin and eosin stain. B, MAP-2. C, synaptophysin. D, chromogranin. E, NSE.

(40\%) of the small cell carcinomas. The intensity of the NSE immunoreactive staining with carcinoid tumors and small cell carcinomas was not as strong as with MAP-2 and synaptophysin. 14 of 25 cases (60\%) of adenocarcinomas and 11 of 25 (44\%) cases of squamous carcinomas were also immunoreactive with NSE.

\section{DISCUSSION}

Lung carcinomas are the leading cause of cancer death for both males and females in United States.
Most pulmonary carcinomas may be readily classified based on their histological features. However, diagnosis and classification by routine light microscopic examination may be difficult and challenging, especially when the tumor is a poorly or undifferentiated carcinoma. Accurate identification of small cell carcinomas in biopsies is critical, because these tumors are optimally treated by modalities other than surgeries. Neuroendocrine tumors generally may be classified into two categories. The first category is an organ-specific group arising from neuroendocrine organs such as pituitary gland, thy- 

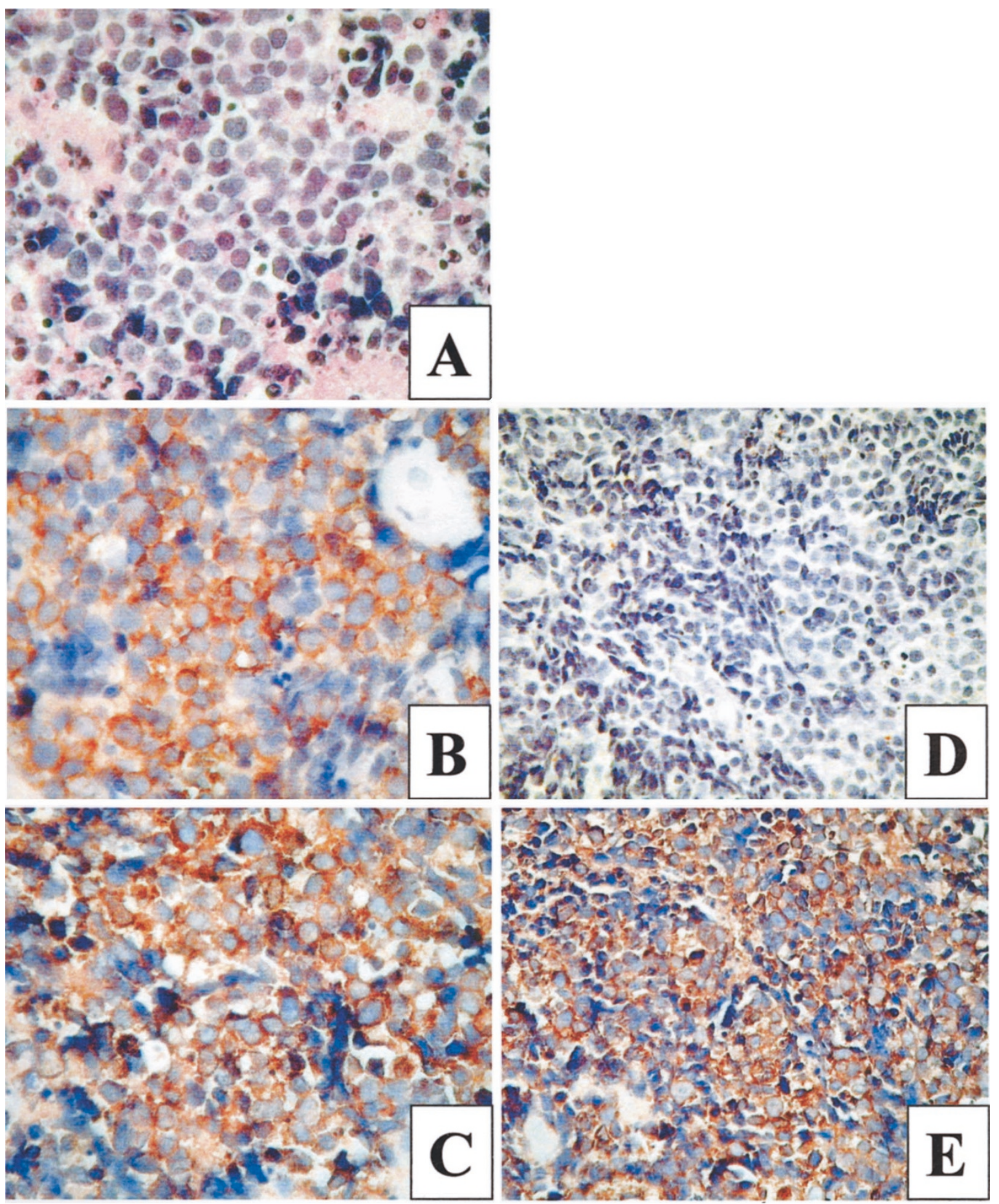

FIGURE 2. Shows immunoreactivity of microtubule-associated protein-2 (MAP-2), synaptophysin, chromogranin, and neuron-specific enolase (NSE) with small cell carcinomas. A, hematoxylin and eosin stain. B, MAP-2. C, synaptophysin. D, chromogranin. E, NSE.

roid, pancreas, and adrenal gland. The second group arises from the diffuse neuroendocrine cells/ Kulchitsky cells that are widely distributed throughout the body and are highly concentrated in the pulmonary and gastrointestinal systems $(9,10,14$, $15,17)$. Neuroendocrine tumors usually demonstrate ultrastructural evidence of dense neuroendocrine secretory granules and express certain specific hormones or proteins that can be detected immunohistochemically $(12,13,16,18,19)$. Synaptophysin, chromogranin, neuron-specific enolase, Leu-7, bombesin, beta-dopamine hydroxylase, and villin have been shown to express immunohistochemically in neuroendocrine neoplasms $(5,6,9-11$, 15, 20-23). Synaptophysin, chromogranin, and neuron-specific enolase are usually included in the immunohistochemical panel to confirm or exclude a neoplasm with neuroendocrine differentiation. 
In this study, we demonstrated that a high percentage of pulmonary carcinoid tumors and small cell carcinomas expressed MAP-2 immunohistochemically. To our knowledge, this is the first study to investigate the utility of MAP-2 expression in pulmonary neuroendocrine neoplasm. We also compared the sensitivity and specificity of MAP-2 immunoreactivity with conventional neuroendocrine markers including synaptophysin, chromogranin, and NSE.

MAP-2, a $280-\mathrm{kD}$ high molecular weight component of MAP family, is expressed in nerve cells and has a characteristic dendritic and somatic subcellular distribution. Ultrastructurally, they are components of filamentous structures that occur between microtubules and other organelles. Neuroscientists have used MAP-2 extensively as a specific and sensitive marker for cells with neuronal differentiation. MAP-2 also has been shown to be expressed in a number of neuronal/neuroblastic neoplasms. Frierson et al. reported that 8 of 11 cases of olfactory neuroblastomas were positive for MAP-2, as well as neurofilaments and synaptophysin. MAP-2 immunoreactivity has also been demonstrated in central neurocytomas, medulloblastomas, gangliogliomas, and dysembryoplastic neuroepithelial tumors (24-26).

Our results indicate that MAP-2 may be used as a sensitive and specific marker for pulmonary carcinoid tumor and small cell carcinoma and that MAP-2 immunohistochemistry can be performed on routine paraffin-embedded formalin-fixed tissue. In this study, MAP- 2 was positive in $100 \%$ of carcinoid tumors and $92 \%$ of small cell carcinomas. The sensitivity and specificity of MAP-2 immunoreactivity with carcinoid tumors and small cell carcinomas is equivalent to synaptophysin and is superior to NSE and chromogranin. Two cases of poorly differentiated squamous carcinomas were focally positive for MAP-2 and synaptophysin. The original diagnoses of poorly differentiated squamous cell carcinomas were based on H\&E examination without confirmation of immunohistochemical studies. Our review of these two cases suggested that they are best classified as large cell carcinomas with neuroendocrine differentiation. These cases suggest that future studies of MAP-2 for the diagnosis of large cell neuroendocrine may be of value. The use of thyroid transcription factor- 1 (TTF-1) in small cell lung carcinoma has been extensively studied. Folpe et al. reported that TTF-1 expression was seen in $35 \%$ of typical carcinoid, $100 \%$ of atypical carcinoids, $75 \%$ of the large cell neuroendocrine carcinomas, and $95 \%$ of small cell carcinomas (27).

The sensitivity of synaptophysin and chromogranin for carcinoid tumor is also $100 \%$. Chromogranin also has the highest specificity, since chromogranin is rarely immunoreactive with any tumors other than carcinoid tumors. Chromogranin is not only a sensitive marker for carcinoid tumors but also has value in separating welldifferentiated neuroendocrine tumors such as a carcinoid tumor from poorly differentiated neuroendocrine tumor such as a small cell carcinoma. In this study, although all 25 cases of carcinoid tumors were always strongly and diffusely positive for chromogranin, 21 of 25 cases of small cell carcinomas were negative for chromogranin. Four of 25 cases of small cell carcinomas showed weak blush immunoreactivity in less than $5 \%$ of the cells.

Our results with conventional immunohistochemical markers of neuroendocrine neoplasms are similar to previous studies $(11,18,28)$. Said et al. examined 9 carcinoid tumors and 12 small cell carcinomas, demonstrating positive staining for chromogranin in all 9 carcinoid tumors and negative staining in all 12 small cell carcinomas (11). Dardick also failed to demonstrate positive staining of chromogranin in 15 cases of small cell carcinomas by using a very sensitive immunohistochemical approach called immunogold electron microscopy (18). Walts et al. also showed absence of staining for chromogranin in 20 cases of small cell carcinomas in cytology specimens (28). However, a few studies have demonstrated that a small proportion of small cell carcinomas are immunohistochemically positive for chromogranin (8). The disparate results of chromogranin immunoreactivity for small cell carcinoma are probably a reflection of the degree of differentiation of the neuroendocrine tumors, which includes the number of the cytoplasmic dense-core neuroendocrine granules in the malignant cells. Because small cell carcinomas are undifferentiated tumors, they may not have any or have very low number of neuroendocrine granules that can be detected by immunohistochemistry. Another explanation for the differing results of chromogranin immunoreactivity for small cell carcinomas is that the positive immunoreactivity in small cell carcinomas may represent area of focal better differentiation in small cell carcinomas.

Synaptophysin is a membrane glycoprotein initially identified from neuronal presynaptic vesicles. SYN has been known as a sensitive and specific marker for tumors with neuroendocrine differentiation. In this study, SYN showed immunoreactivity with $100 \%$ of carcinoid tumors, $92 \%$ of small cell carcinomas, $16 \%$ of adenocarcinomas, and $8 \%$ of squamous cell carcinomas. The sensitivity and specificity of SYN immunoreactivity with neuroendocrine tumors is the same as MAP-2.

Enolase is a glycolytic enzyme composed of dimers of three subunits named alpha, beta, and gamma subunits. The gamma-gamma dimers of enolase are found mainly in neurons and neuroendocrine cells and are referred to neuron-specific 
enolase. However, gamma subunits have been found in other cells as well. Although NSE has been used as a marker for neuroendocrine tumors, we as well as other have demonstrated that NSE is nonspecific for the diagnosis of neuroendocrine tumors. In this study, NSE showed immunoreactivity in $60 \%$ of adenocarcinomas and $44 \%$ of squamous carcinomas. Loy et al. also showed immunoreactivity of NSE with $60 \%$ of carcinomas lacking neuroendocrine differentiation.

In conclusion, we believe that MAP-2 is a very sensitive and specific marker for the pulmonary tumors of neuroendocrine differentiation. We recommend that MAP-2 be added to immunohistochemical panels to separate non-neuroendocrine from neuroendocrine lung tumors.

\section{REFERENCES}

1. Avila J. Microtubule function. Life Sci 1991;327-34.

2. Maccioni RB, Cambiazo V. Role of microtubule-associated proteins in the control of microtubule assembly. Physiol Rev 1995;835-64.

3. Addis B, Hamid Q, Ibrahim NB, Fahey M, Bloom SR, Polak JM. Immunohistochemical markers of small cell carcinoma and related neuroendocrine tumors of the lung. J Pathol 1987;153:137-50.

4. Brambilla E, Veale D, Moro D, Morel F, Dubois F, Brabbilla C. Neuroendocrine phenotype in lung cancers. Am J Clin Pathol 1992;98:88-97.

5. Gould VE, Lee I, Wiedenmann B, Moll R, CHejfec G, France WW. Synaptophysin: a novel marker for neurons, certain neuroendocrine cells, and their neoplasms. Hum Pathol 1986;17:979-83.

6. Graziano IP. Expression of neuron-specific enolase, chromogranin A, synaptophysin and Leu-7 in lung cancer cell lines. J Exp Clin Cancer Res 1988;17:165-73.

7. Jensen S, Gazdar AF, Cuttitta F, Russell EK, Linnoila RI. A comparison of synaptophysin, chromogranin, and L-dopa decarboxylase as markers for neuroendocrine differentiation in lung cancer cell lines. Cancer Res 1990;50:6068-74.

8. Kiriakogiani-Psaropoulou P, Malamou-Mitsi V, Martinopoulou U, Legaki S, Tamvakis N, Vrettou E, et al. The value of neuroendocrine markers in non-small cell lung cancer: a comparative immunohistopathologic study. Lung Cancer 1994;11:353-64.

9. Loy TS, Darkow GVD, Quesenberry JT. Immunostaining in the diagnosis of pulmonary neuroendocrine carcinomas. An immunohistochemical study with ultrastructural correlations. Am J Surg Pathol 1995;19:173-82.

10. Poola I, Graziano SL. Expression of neuron-specific enolase, chromogranin A, synaptophysin and Leu-7 in lung cancer cell lines. J Exp Clin Cancer Res (Italy) 1998;17:165-73.
11. Said JW, Vimadalal S, Nash G, Shintaku P, Heusser R, Sasson $\mathrm{AF}$, et al. Immunoreactive neuro-specific enolase, bombesin and chromogranin as marker for neuroendocrine lung tumors. Hum Pathol 1985;16:236-40.

12. Haimoto H, Takehashi Y, Koshikawa T, Nagura H, Kato K. Immunohistochemical localization of gamma-enolase in normal human tissue other than nervous and endocrine tissue. Lab Invest 1985;52:257-63.

13. Lee I, Gould VE, Moll R, Wiedenmann B, Franke WW. Synaptophysin expressed in the bronchopulmonary tract: neuroendocrine cells, neuroepithelial bodies, and neuroendocrine neoplasms. Differentiation 1987;34:115-25.

14. Gaffey MJ, Mills SE, Lack EE. Neuroendocrine carcinoma of the colon and rectum. Am J Surg Pathol 1990;14:1010-23.

15. Wick MR, Berg LC, Hertz MI. Large cell carcinoma of lung with neuroendocrine differentiation- a comparison with large cell undifferentiated pulmonary tumors. Am J Clin Pathol 1992;97:796-805.

16. Yousem SA. Pulmonary carcinoid tumors and welldifferentiated neuroendocrine carcinomas-is there room for an atypical carcinoid? Am J Clin Pathol 1991;95:763-4.

17. Zhang PJ, Harris KR, Alobeid B, Brooks JJ. Immunoexpression of villin in neuroendocrine tumors and its diagnostic implications. Arch Pathol Lab Med 1999;123:812-6.

18. Dardick I, Christensen H, Stratis M. Immunoelectron microscopy for chromogranin A in small cell neuroendocrine carcinoma of lung. Ultrastruct Pathol 1996;20:361-8.

19. Silver MM, Lines LD, Hearn SS. Immunogold detection of chromogranin A in the neuroendocrine tumor. Ultrastruct Pathol 1993;17:83-92.

20. Linnoila RI, Mulshine JL, Steinberg SM, et al. Neuroendocrine differentiation in endocrine and nonendocrine lung carcinomas. Am J Clin Pathol 1988;90:641-52.

21. Michels S, Swonson PE, Wick MR. Leu-7 in small cell neoplasms-an immunohistochemical study with ultrastructural correlations. Cancer 1987;60:2958-64.

22. Warren WH, Faber LP, Gould VE. Neuroendocrine neoplasm of the lung. J Thorac Cardiovasc Surg 1989;98:321-32.

23. Ekiksson B, Arnberg H, Hellman U, Lundqvist G, Wernstedt C, Wilander E. Chromogranins—-new sensitive markers for neuroendocrine tumors. Acta Oncol 1989;28:325-9.

24. Frierson HF Jr, Ross GW, Mills SE, Frankfurter A. Olfactory neuroblastomas. Additional immunohistochemical characterization. Am J Clin Pathol 1990;94:547-53.

25. Hessler RB, Lopes MB, Frankfurter A, Vandenbergg SR. Cytoskeletal immunohistochemistry of central neurocytomas. Am J Surg Pathol 1992;16:1031-8.

26. Yasha TC, Mohanty A, Radhesh S, Santosh V, Das S, Shankar SK. Infratentorial dysembryoplastic neuroepithelial tumor (DNT) associated with Arnold-Chiari malformation. Clin Neuropathol (Germany) 1998;17:305-10.

27. Folpe AL, Gown AM, Lamps LW, Garcia R, Dail DH, Zarbo RJ, et al. Thyroid transcription factor-1: immunohistochemical evaluation in pulmonary neuroendocrine tumors. Mod Pathol 1999;12:5-8.

28. Walts AE, Said JW, Shintaku P. Chromogranin as a marker of neuroendocrine cells in cytologic material-an immunocytochemical study. Am J Clin Pathol 1984;84:273-7. 